\title{
Influencing Fatty Acids Composition of Yeasts by Lanthanides
}

\author{
Irena Kolouchova, Tomas Rezanka, Michal Zimola \\ Department of Biotechnology, University of Chemistry and technology Prague \\ Technicka 5, Prague, Czech Republic \\ irena.kolouchova@vscht.cz; rezanka@biomed.cas.cz; michalzimola@gmail.com
}

\section{Extended Abstract}

The growth of microorganisms is affected by cultivation conditions, concentration of carbon and nitrogen sources and the presence of trace elements. One of new possibilities of influencing the production of biomass or lipids is the use of lanthanides. Lanthanides are biologically non-essential elements with wide applications in technology and industry and their concentration as environmental contaminants is therefore increasing. Although non-essential, lanthanides have been proposed (and even used) to produce beneficial effects in plants but their mechanisms of action are unclear. Recently, it was suggested that they may replace essential elements. We tested the effect of low concentrations of lanthanides on traditional biotechnologically useful yeast species (Kluyveromyces polysporus, Saccharomyces cerevisiae, Torulospora delbrueckii), and species capable of high accumulation of lipids (Rhodotorula glutinis, Trichosporon cutaneum, Candida sp., Yarrowia lipolytica). Growth characteristics were determined in the above yeast strains cultivated with different lanthanides or monazite (calculated to lanthanum proportional content) concentrations $(0.03 ; 0.3 ; 1 ; 3 \mathrm{mg} / \mathrm{L})$. The cultivations were carried out for $96 \mathrm{~h}$ (until early stationary phase), cultivation temperature was $30{ }^{\circ} \mathrm{C}$. The fatty acids extraction was based on the method of Bligh and Dyer (1959). Gas chromatography-mass spectrometry of fatty acid picolinyl esters was done on a GC-MS system. The lanthanides in selected concentration did not have any significant inhibitory effect on yeast growth and some of them even showed a stimulatory effect. Low concentrations of some lanthanides were conducive to an increase in biomas and also higher production of palmitoleic acid, commonly used in cosmetics and medicine, and $\omega 6$-linoleic acid which is a precursor of thromboxanes, prostaglandins and leucotrienes. Lanthanum enhanced the cell biomass of $\mathrm{T}$. cutaneum by as much as $150 \%$ and other individual selected lanthanides (lanthanum, praseodymium, gadolinium and monazite as a naturally occurring mixture of lanthanides) in the range of 23 and $57 \%$. Cultivation with monazite increased the cell biomass in Candida sp., R. glutinis and T. cutaneum by $30-40 \%$ and in $Y$. lipolytica by $60 \%$. A sizable increase in lipid content occurred upon addition of praseodymium and lanthanum to K. polysporus (from $5 \%$ to $9 \%$ ), Candida sp. (from $35 \%$ to $43 \%$ ), Y. lipolytica (from $18 \%$ to $25 \%$ ) and a 10-fold higher concentration of monazite $(3 \mathrm{mg} / \mathrm{l})$ boosted the increase of lipid content by some $50 \%$ compared to the effect of $0.3 \mathrm{mg} / \mathrm{l}$. The highest increase of the palmitoleic and linoleic acid content was brought about by lanthanum, and in some strains by monazite irrespective of its concentration. The highest yield of palmitoleic and linoleic acid, in excess of $300 \mathrm{mg} / \mathrm{g}$, was obtained by culturing $T$. cutaneum with lanthanum and monazite. 